\title{
La educación sexual: un desafío para la educación católica ${ }^{1}$
}

Luz Eugenia Montero-Ossandón

orcid.org/0000-0003-4405-0731

Pontificia Universidad Católica

de Chile, Chile

lmontero@uc.cl

\section{Angelina María Dois-Castellón}

orcid.org/00oo-0o03-2978-6644

Pontificia Universidad Católica

de Chile, Chile

adois@uc.cl

\section{Carmen Aída Domínguez-Hidalgo}

Pontificia Universidad Católica

de Chile, Chile

cadoming@puc.cl

\section{Paz Valverde-Forttes}

orcid.org/0000-0002-4518-4822 Universidad de los Andes, Chile pvalverd@uc.cl

\section{Mauricio Bicocca-Gino}

orcid.org/000o-0003-1267-9033 Universidad de los Andes, Chile mbicocca@uandes.cl

\section{Resumen}

En el mundo, la educación sexual en colegios católicos constituye un desafío. Desde el 2010, en Chile existe una ley que obliga a todos los colegios a contar con un programa de educación sexual. Sin embargo, su implementación en colegios de identidad católica ha sido poco evaluada. El artículo explora las dificultades y desafios percibidos por los docentes a cargo de la educación sexual en colegios católicos de la Región Metropolitana de Santiago de Chile.

Método: diseño cualitativo con datos recolectados con entrevistas semi-estructuradas, a partir de un guion temático sobre una muestra intencionada. Se realizó análi-

1 Proyecto financiado por Vicerrectoría de Investigación y Dirección de Pastoral y Cultura Cristiana, Pontificia Universidad Católica de Chile. Las entidades financiadoras no participaron directamente ni influyeron de alguna forma en el diseño, recolección, análisis de resultados, redacción y revisión del manuscrito.

Recepción: 15-09-2015 | Envío a pares: 26-07-2016 | Aceptación por pares: 13-06-2017 | Aprobación: 14-06-2017 
ISSN 0123-1294 | e-ISSN 2027-5358 | Educ.Educ. Vol. 20. No. 3 | Septiembre-diciembre de 2017 | pp. 343-363.

Universidad de La Sabana | Facultad de Educación

sis descriptivo y relacional, basado en los procedimientos de la Grounded Theory. El criterio de finalización fue la saturación teórica de los datos.

Las principales dificultades encontradas en la educación sexual en colegios católicos son: la tensión entre los postulados de la doctrina católica, las vivencias de los jóvenes, un entorno erotizado y adverso a los valores cristianos y las creencias de las familias y de los profesores. Además, la falta de participación de las familias y la dificultad operativa para impartir esta enseñanza, por la falta de tiempo protegido, de infraestructura adecuada y de profesores capacitados.

\section{Palabras clave}

Educación sexual; política educativa; educación católica; evaluación escolar; Santiago de Chile (Fuente: Tesauro de la Unesco). 


\title{
Sex Education: A Challenge for Catholic Education
}

\begin{abstract}
Sex education in Catholic schools the world over poses a challenge. Since 2010, all schools in Chile have been required by law to offer a sex education program. However, little has been done to evaluate its implementation in schools that identify as Catholic. The article explores the difficulties and challenges perceived by teachers who are in charge of sex education at Catholic schools in the metropolitan area of santiago de Chile.

Method: A qualitative design was used and data was collected through semi-structured interviews, based on a thematic script concerning an intentional sample. A descriptive and relational analysis was carried out, using grounded theory procedures. Theoretical saturation of the data was the criterion for completion.

The main difficulties found with sex education in Catholic schools are: the tension between the postulates of Catholic doctrine, the experiences of young people, an environment that is erotic and adverse to Christian values; and the beliefs of families and teachers. In addition, there is a lack of participation on the part of families and the operational difficulty of imparting education of this type, due to want of protected time, adequate infrastructure and trained teachers.
\end{abstract}

\section{Keywords}

Sex education; education policy; Catholic education; school evaluation; Santiago de Chile (Source: Unesco Thesaurus). 


\section{A educação sexual: um desafio para a educação católica}

\section{Resumo}

Em todo o mundo, a educação sexual em colégios católicos constitui um desafio. Desde 2010, no Chile, existe uma lei que obriga os colégios a contarem com um programa de educação sexual. Contudo, sua implantação em colégios de identidade católica tem sido pouco avaliada. Este artigo explora as dificuldades e desafios percebidos pelos docentes responsáveis pela educação sexual em colégios católicos da região metropolitana de Santiago do Chile.

Método: desenho qualitativo, com dados coletados em entrevistas semiestruturadas, a partir de um roteiro temático sobre uma amostra intencionada. Realizou-se análise descritiva e relacional, baseada nos procedimentos da Grounded Theory. O critério de finalização foi a saturação teórica dos dados.

As principais dificuldades encontradas na educação sexual em colégios católicos são: a tensão existente nos que professam o catolicismo, as vivências dos jovens, o ambiente erotizado e adverso aos valores cristãos e crenças das famílias e dos professores. Além disso, a falta de participação das famílias e a dificuldade operativa para ensinar sobre o tema, a falta de tempo protegido, de infraestrutura adequada e de professores capacitados.

\section{Palavras-chave}

Avaliação escolar; educação católica; educação sexual; política educativa; Santiago do Chile (Fonte: Tesauro da Unesco). 
El sistema educativo chileno se organiza en dos etapas. La primera es de educación básica obligatoria y considera ocho grados anuales, y la segunda es de educación media, no obligatoria, y considera cuatro grados más, es decir que un estudiante realiza en promedio una formación escolar de doce años. En este sistema existen distintos tipos de establecimientos educacionales según sea su dependencia administrativa y financiera. Por un lado, se encuentran los colegios de administración municipal, los cuales son de propiedad y financiamiento estatal. Estos corresponden al mayor número y poseen la mayor cantidad de matrículas. Por otra parte, existen los colegios privados con subsidio, de propiedad y administración de particulares, pero que reciben una subvención estatal por cada alumno matriculado. Finalmente, están los colegios privados, cuya propiedad y administración pertenece a particulares y el financiamiento procede de las familias de los alumnos. Estos son los que poseen la menor matrícula (Madero, 2012). Del total de establecimientos, los colegios católicos suman 846 a lo largo de todo el país, ya sea de tipo privado, con o sin subsidio, que llegan a cubrir el $16,8 \%$ de la matrícula total nacional y el $27,2 \%$ de toda la educación particular, con 596.586 alumnos/as al año 2012 (Madero, 2012).

Uno de los grandes desafíos de la educación escolar actual es la educación sexual. Para ello se han establecido políticas públicas orientadas a mejorar la formación en esta materia, promoviendo la implementación de programas de educación sexual. Para ello el gobierno ha puesto a disposición de los establecimientos educacionales siete programas de educación sexual, con lo cual aspira a disminuir la tasa de embarazo adolescente, retrasar el inicio de la actividad sexual en los jóvenes y formar e informar a los alumnos a fin de promover la responsabilidad y libertad en sus decisiones (Ministerio de Educación, 2012). Si bien estos programas son diferentes entre sí, ninguno de ellos tiene como enfoque explícito la antropología cristiana. Los colegios son libres de escoger cualquiera de estos o de desarrollar un programa propio, consecuente con su línea educativa.
En este contexto, el objetivo de este estudio es explorar las dificultades y desafíos percibidos por docentes y orientadores a cargo de programas de educación sexual en colegios católicos de la Región Metropolitana de Chile. A partir de esto, el escrito se articula en los siguientes ejes: marco referencial, material y método, resultados, discusión, conclusión.

\section{Marco referencial}

\section{Perspectiva legal y de política pública en relación con la educación en Chile}

Desde la perspectiva legal, la existencia de colegios religiosos y laicos se funda en el ordenamiento jurídico chileno, que asegura que toda persona tiene el derecho a tener una educación conforme a sus creencias. De ahí que la Constitución del país consagra, entre sus garantías, específicamente en el artículo 19, No. 11, que "la libertad de enseñanza incluye el derecho de abrir, organizar y mantener establecimientos educacionales", complementado luego, de modo principal, primero por la Ley sobre Constitución Jurídica de las Iglesias y Organizaciones Religiosas (Ley 19638 de 1999) que establece en su artículo $6 \mathrm{~d}$ que uno de los contenidos de la libertad religiosa y de culto de toda persona consiste en "recibir e impartir enseñanza o información religiosa por cualquier modo, elegir para sí y los padres para los menores no emancipados y los guardadores para los incapaces bajo su tuición y cuidado la educación religiosa y moral que esté de acuerdo con sus propias convicciones".

Este derecho a la libertad se les reconoce a los denominados fundadores, dueños o sostenedores de los establecimientos educativos y además a los padres. A los primeros, en cuanto les concede la facultad recién referida de crear establecimientos educacionales. Por su parte, los padres son titulares de derechos educativos como resulta de modo nítido del inciso 4 del artículo 19, No. 11, que señala que "los padres tienen el derecho de escoger el establecimiento 
ISSN 0123-1294 | e-ISSN 2027-5358 | Educ.Educ. Vol. 20. No. 3 | Septiembre-diciembre de 2017 | pp. 343-363.

Universidad de La Sabana | Facultad de Educación

de enseñanza para sus hijos". El significado de esta libertad tiene como elemento esencial "el derecho de abrir, organizar y mantener establecimientos educacionales" (Bertelsen, 2004, p. 167), facultades cuyo contenido ha sido desarrollado por el Tribunal Constitucional al precisar que ellas implican el derecho a crear esos establecimientos de acuerdo

[...] con el ideario del proyecto educativo de los fundadores respectivos. En seguida, queda asegurado el derecho de organizarlos o determinar, los fundadores o quienes les sigan, las caracteristicas del establecimiento en nexo con sus finalidades u objetivos y métodos para lograrlos; rasgos típicos de la docencia y de los profesionales que la lleven a cabo; régimen de dirección, administración y responsabilidad; reglas pertinentes al orden, disciplina en la convivencia interna; sistema financiero o vínculos con otras instituciones. Por último, la libertad de enseñanza incluye la facultad de mantener, esto es, conservar o sostener el establecimiento en el tiempo, modificando su organización o, en última instancia, cerrarlo o transferirlo a terceros².

Esta precisión, como bien se ha resaltado, debe ser tomada como una verdadera definición de lo que, en nuestro sistema, alcanzan esas tres facultades inherentes a la libertad de enseñanza (Bertelsen, 2004, p. 168).

De este modo, tanto los establecimientos educacionales católicos tienen el derecho a impartir una educación conforme a su ideario como los padres tienen el derecho a elegir y recibir de los mismos una educación, en todas las áreas que ella comprende, conforme a la fe que profesan para sus hijos. En este sentido, temas vinculados a los valores procesados por la comunidad escolar se transforman en ele-

2 Tribunal Constitucional. Sentencia de 14 de junio de 2004 redactada por los ministros Eugenio Valenzuela Somarriva y José Luis Cea Egaña, con prevención y voto particular del ministro Juan Agustín Figueroa Yávar. mentos discrecionales al decidir el tipo de establecimiento educacional.

Uno de los ámbitos que comprende la educación es, en el presente, el de la sexualidad. En efecto, la regulación de la fertilidad constituye una política del Ejecutivo que data de 1967 y que ha derivado en múltiples políticas públicas orientadas a la educación sexual (Ministerio de Salud, 2013), por lo demás, precedidas de un amplio debate público en torno a sus contenidos. Así sucedió en 1996 ante la propuesta de Jornadas de Conversación sobre Afectividad y Sexualidad entre profesores y alumnos durante ciertos días de la semana, las que fueron cuestionadas por amplios sectores, entre ellos, por la Iglesia Católica, en razón de plantearse sin un marco de valores adecuado. Más tarde, el Ministerio de Salud dictó las denominadas Normas Nacionales de Fertilidad que comprendían el derecho de toda persona a recibir información en materia de regulación de la fertilidad, las que el año 2007 serían declaradas inconstitucionales por el Tribunal Constitucional. Esta normativa fue nuevamente dictada por el Ejecutivo mediante Decreto Supremo e igualmente declarada inconstitucional por el mismo Tribunal en lo que concierne a la entrega de la denominada "anticoncepción de emergencia” en el sistema público de salud y a la entrega de información sobre la misma en el ámbito de cualquier consejería sobre regulación de la fertilidad. Posteriormente, en el año 2010 se dictó la Ley 20418 que fija las Normas sobre Información, Orientación y Prestaciones en materia de regulación de la fertilidad, la cual establece el derecho de toda persona "a recibir información y orientación completa en materia de regulación de la fertilidad" (art. 1), disponiendo que los establecimientos educacionales reconocidos por el Estado deben incluir dentro del ciclo de enseñanza media "un programa de educación sexual, el cual, según sus principios y valores, incluya contenidos que propendan a (sic) una sexualidad responsable e informe de manera completa sobre los diversos métodos de regulación de la fertilidad existentes y autorizados", ello de 
acuerdo con el "proyecto educativo, convicciones y creencias de cada establecimiento educacional, en conjunto con los centro de padres y apoderados" (Gobierno de Chile, 2012). Al amparo de esta ley, el Ministerio de Educación, en conjunto con el Servicio Nacional de la Mujer, puso a disposición de los establecimientos educacionales siete programas de educación sexual, con lo que aspiraba a disminuir la tasa de embarazo adolescente, retrasar el inicio de la actividad sexual en los jóvenes y formar e informar a los estudiantes a fin de promover la responsabilidad y libertad en sus decisiones (Ministerio de Educación, 2012). Estos programas difieren en sus propósitos, en las antropologías que los sustentan y en las metodologías, y ninguno de ellos tiene un enfoque explícito basado en la antropología cristiana. Sin embargo, los colegios son libres de escoger entre ellos o de desarrollar un programa propio, consecuente con su línea educativa.

\section{Programas de educación sexual desde una perspectiva cristiana. Antecedentes}

En el marco de la presente investigación, hay que señalar que la educación sexual en los colegios católicos constituye hasta el presente un gran desafío mundial. Este problema es prioritario en el campo de la educación católica, a saber, el de la identidad de los colegios católicos (Sultmann y Brown, 2014). Una revisión de la literatura científica sobre el tema de la educación sexual desde una perspectiva católica permite afirmar que son escasos los trabajos que enfrentan la temática para su indagación, ya sea por la dificultad del problema y los múltiples detalles que el mismo conlleva, o por la falta de programas concienzudamente implementados y posteriormente evaluados, o por los problemas que en los últimos años han conmocionado la opinión pública respecto de abusos sexuales, o porque se ha preferido no investigar sobre esta materia. Más allá de cuáles sean las razones, debe subrayarse que este vacío de conocimientos es un hecho innegable, y no puede dejar de reconocerse que la educación sexual, desde una perspectiva católica, es una cuestión crucial para los colegios confesionales. La doctrina no puede quedarse en una mera discusión de ideas, sino que debe asumir los retos de la práctica (McKinney y Sullivan, 2013),

Los programas de educación sexual basados en principios religiosos han ganado cierta notoriedad en los recientes años. En este sentido, programas como True Love Waits, de orientación cristiana (Whitehead, 2001) de los Estados Unidos de Norteamérica, en el cual los adolescentes toman votos de castidad hasta el matrimonio, han sido particularmente exitosos en cuanto que han bajado los índices de actividad sexual de los adolescentes hasta ese momento (Rector, 2002). Otras investigaciones sugieren que este tipo de programas donde la conducta está guiada solo por el principio de la abstinencia muestran que, si bien se posterga la edad para la iniciación sexual, las personas que rigen su conducta así tienen altos riesgos de embarazo y de adquirir enfermedades de transmisión sexual (Bruckner y Bearman, 2005).

A partir de lo antes descrito, cabe preguntar por qué centrarse en investigar programas de educación sexual basados en una perspectiva religiosa. Hay varias razones que justifican el interés investigativo en este problema. Primero, la religión es ciertamente un factor de socialización en los adolescentes, a veces de mayor peso que el de los padres y los compañeros, y ha recibido escasa atención investigativa respecto de los efectos de su influencia en las conductas sexuales de los jóvenes (Freedman-Doan, Fortunato, Henshaw y Titus, 2013; Regnerus, 2007). Segundo, la religión crea comunidades cuyos miembros comparten valores que influyen directamente en sus conductas y en donde la espiritualidad entrega una visión trascendente (no física) de la vida que otorga sentido a la existencia, lo que afecta la toma de decisiones y el comportamiento (Luquis, Brelsford y Rojas-Guyler, 2011; Peterson y Seligman, 2004). Tercero, la dimensión sexual es particularmente relevante para la religión; de hecho, todas las religio- 
nes tradicionales contienen esta dimensión en sus doctrinas (Regnerus, 2007).

En el caso concreto de la Iglesia Católica, la familia es la primera, pero no la única y exclusiva comunidad educadora de los hijos. Los padres tienen el derecho y el deber de educar cristianamente a sus hijos, en lo que constituye la llamada "iglesia doméstica". Sin embargo, la familia cristiana requiere de un acompañamiento adecuado en esta labor, a través tanto de la catequesis en las parroquias como de la enseñanza de la religión y la moral en los colegios de inspiración católica. La escuela debiera ser la expansión natural de la tarea educativa de la familia, a través del fomento del encuentro con Cristo, sin desconocer el respeto por la competencia del Estado para promulgar normas generales sobre instrucción (Benedicto XVI, 2009, 2008; Juan Pablo II, 1981).

En el ámbito de la educación sexual, la doctrina católica establece que esta debiera realizarse bajo la supervisión de los padres, tanto en casa como en los centros educativos escogidos por ellos. Se espera que quienes desempeñan una actividad educadora contribuyan a la formación en torno al amor humano y a la sexualidad, preparando a los jóvenes para vivir la castidad, aquella virtud que desarrolla la auténtica madurez de la persona y que hace capaz de respetar y promover el "significado esponsal" del cuerpo. En ese sentido, la Iglesia se opone a un sistema de información sexual separado de los principios morales (Juan Pablo II, 1985, 1981; Pablo VI, 1968; Wojtyla, 1960; Pío XI, 1930).

\section{Percepciones de docentes y orientadores cristianos a cargo de programas de educación sexual}

Actualmente, desde una perspectiva cristiana, la educación sexual es un desafío para los docentes y los orientadores. Existen al respecto dificultades que surgen de múltiples ámbitos; por ejemplo, el lenguaje utilizado, las formas de enfrentar la problemática, los contenidos, el tiempo requerido, etc.
(Aschraft, 2011). Asimismo, la percepción del valor que tiene un programa por parte de los docentes es central para su efectiva implementación (Kirby, 2002; Stein y Wang, 1988). Distintos estudios han identificado ciertos factores que influyen en las disposiciones para la enseñanza efectiva de una educación sexual desde una perspectiva cristiana, tales como: 1) los conocimientos que se poseen sobre la materia en cuestión; 2) el nivel de conformidad con los principios que se enseñan; 3) el entrenamiento en la enseñanza de dicha materia; 4) el grado de vivencia existencial del docente con la enseña impartida, es decir: la coherencia de vida (Cohen, Byers y Sears, 2012). Estos elementos afectan y están directamente relacionados con la percepción que tienen los docentes a cargo de programas de educación sexual desde una perspectiva cristiana y con el grado de efectividad que puede alcanzar su enseñanza en esa materia.

\section{Material y método}

\section{Diseño, universo y muestra}

Este es un estudio cualitativo de diseño analítico relacional, basado en la Teoría Fundamentada (Glaser y Strauss, 2010; Strauss y Corbin, 1998; Patton, 1990), cuyo universo estuvo formado por colegios que cumplieran los siguientes criterios de inclusión: a) colegio católico (privado sin y con subsidio) y b) poseer al menos una sede en la Región Metropolitana. Para acceder a los participantes se utilizó un muestreo por conveniencia y en cadena, intentando respetar los criterios de representatividad, heterogeneidad y accesibilidad (Sandoval, 1996).

Para la recolección de la información se realizaron, entre abril y noviembre del 2013, entrevistas semiestructuradas basadas en un guion temático a los encargados del programa de educación sexual ("orientador"). Cabe destacar que el encargado no siempre fue el orientador y que también se realizaron entrevistas en las que se sumaron otras autoridades del colegio (director, coordinador y/o soste- 
nedor económico). Las entrevistas fueron realizadas por ayudantes de investigación capacitados o por miembros del equipo de investigación. El criterio de finalización de las entrevistas fue la saturación teórica de la información (Flick, 2004). Cada entrevista tuvo una duración aproximada de una hora, fue grabada en su totalidad y transcrita verbatim.

\section{Análisis de datos}

El análisis se realizó bajo la aplicación sistemática del método y los procedimientos propuestos por la Grounded Theory (Glaser y Strauss, 2010; Strauss y Corbin, 1998). Se utilizó codificación abierta para identificar los conceptos emergentes, sus propiedades y dimensiones. Una vez terminado el análisis de la primera entrevista, se procedió a realizar la segunda y, siguiendo el mismo procedimiento anterior, se procedió a codificarla. A la luz de dichas codificaciones, se hicieron algunas modificaciones en el guion de entrevista para profundizar en algunos temas, procedimiento que se repitió con el análisis de las siguientes entrevistas.

Para el componente analítico-relacional, se utilizó codificación axial para referirse a categorías y subcategorías en torno a ejes aglutinadores y codificación selectiva para integrar las categorías principales a un esquema teórico más amplio que per- mitiera formular un modelo explicativo (Flick, 2004). Para disminuir el sesgo, las categorías relevantes fueron determinadas según los resultados aportados por las entrevistas, los antecedentes bibliográficos y la triangulación por expertos. Las consideraciones éticas de este estudio se originan a partir del resguardo de la dignidad de la persona durante toda la investigación, considerando el consentimiento informado aprobado por el Comité de Ética en Investigación de la Facultad de Educación de la Pontificia Universidad Católica de Chile, que asegura la confidencialidad de la información, la participación voluntaria y el respeto por la autonomía de las personas participantes.

\section{Resultados}

La muestra quedó conformada por ocho (8) colegios, cuyas características se muestran en la Tabla 1.

\section{Análisis relacional}

Los códigos obtenidos en el análisis descriptivo (codificación abierta) se reagruparon según dos aspectos que se destacan en los resultados (codificación axial). Una segunda etapa (codificación selectiva) permitió construir un modelo comprensivo que articula los aspectos esenciales de los resultados con el fenómeno estudiado. Del análisis relacional se

Tabla 1. Distribución de la muestra

\begin{tabular}{|c|c|c|c|c|}
\hline Colegio & Tipo de financiamiento & Nivel socioeconómico & Sistema & Filiación \\
\hline 1 & Privado & Alto & Coeducacional & Congregación \\
\hline 2 & Privado con subsidio & Medio & Mixto & Congregación \\
\hline 3 & Privado & Alto & Femenino & Congregación \\
\hline 4 & Privado & Alto & Masculino & Sin filiación específica \\
\hline 5 & Privado & Medio & Mixto & Congregación \\
\hline 6 & Privado con subsidio & Medio-bajo & Mixto & Sin filiación especifica \\
\hline 7 & Privado & Alto & Masculino & Congregación \\
\hline 8 & Privado con subsidio & Medio-bajo & Femenino & Congregación \\
\hline
\end{tabular}

Fuente: Los autores. 
desprenden dos elementos principales que estarían influyendo en los programas de educación sexual de los colegios católicos participantes en el estudio: 1) influencia del contexto: la antropología cristiana, las políticas públicas y el entorno de niños y jóvenes; 2 ) personas involucradas en su ejecución: los alumnos, las familias y los profesores.

\section{Influencia del contexto}

Respecto del contexto, se distinguen tres aspectos que impactan en el desarrollo de los programas de educación sexual escolar. El primero de ellos se vincula con la antropología cristiana y la doctrina de la Iglesia Católica en relación con la sexualidad, el matrimonio y la familia (Juan Pablo II, 1981). Esta doctrina promueve la castidad, la postergación de las relaciones sexuales hasta el matrimonio y la vivencia de las relaciones sexuales matrimoniales en comunión y abiertas a la fecundidad, lo que, según los entrevistados, es puesto en tensión permanente por las políticas públicas y por el entorno en que se desarrollan los estudiantes.

Los entrevistados describen el medio en que se insertan los estudiantes como un mundo erotizado que estimula la promiscuidad y el inicio cada vez más precoz de las relaciones sexuales y que, a su vez, relega la sexualidad a una aproximación vinculada solo al placer, donde además predominan valores tales como el individualismo, el relativismo y la inmediatez. Los entrevistados perciben que los alumnos tienen mucho más conocimiento que antes en temas de sexualidad, dada la facilidad para acceder a información y a contenidos de carácter sexual. Esto hace que muchas veces se enfrenten a información contradictoria entre lo que se transmite en los establecimientos educacionales y lo que ven en los medios de comunicación.

Tenemos en contra todo el ambiente. Todo el ambiente está en contra de estos valores. Estamos en estos momentos en un mundo absolutamente erotizado, los alumnos reciben un bombardeo permanente, permanente, permanente desde que salen de aquí del colegio de todo lo contrario, o sea, de pasarlo bien, de que el sexo es algo para gozar, que el sexo es algo para disfrutar, que da lo mismo que yo use al otro y después lo deseche como quien desecha un zapato viejo. $\left(E_{3}, 165-170\right)$

Las características antes descritas, entre otros aspectos, han potenciado el desarrollo de políticas públicas en materia de educación sexual y de salud sexual y reproductiva, orientadas a la prevención de las enfermedades de transmisión sexual y del embarazo adolescente, principalmente a través del uso de métodos anticonceptivos de barrera desde la primera relación sexual. Este elemento se constituye, a juicio de los entrevistados, como el segundo aspecto que incide en las decisiones que toman los colegios respecto de sus programas de educación sexual. Por una parte, la oferta ministerial no considera programas completamente alineados con los valores y principios de la doctrina cristiana y, por otra, algunos de ellos tienen además dificultades de implementación, razón por la cual los colegios prefieren optar por crear programas nuevos o adaptar los ya existentes, porque se "necesita mucha capacitación, [...] y la rotación de profesores es altísima, por lo tanto, tú capacitabas y se te iban. Después había otro factor que es el de que es muy alejado de la realidad de los niños del tipo de colegio donde trabajo yo" (E5, 18-21).

Por último, los entrevistados explican que el Ministerio de Educación no considera horas para la ejecución de los programas de educación sexual ni recursos humanos y materiales para capacitar a los profesores, lo que dificulta la implementación de la política pública vigente en los establecimientos educacionales. El análisis de las entrevistas permitió establecer que las instituciones educacionales optan entonces por dos modalidades para impartir educación sexual. La primera no considera un programa formal y estructurado, en el que los contenidos que se entregan nacen de las necesidades de los 
estudiantes en función de las temáticas abordadas y en determinados programas de cursos, particularmente en los de ciencias (con un enfoque biológico) y en los de religión. Asimismo, el tema de la educación sexual se aborda incidentalmente ante situaciones específicas que ocurren en la comunidad escolar, como el embarazo de alguna estudiante. Solo en algunos casos, ante la emergencia de una problemática en algún curso, el equipo de psicología y orientación del colegio diseña una unidad de trabajo que implementa el profesor jefe durante la hora de Orientación (sin recibir capacitación para ello ni en el contenido ni en la metodología). Los entrevistados reconocen que los profesores no cuentan con espacios protegidos dentro de su jornada para dialogar, consensuar e implementar el tratamiento del tema, y en algunos casos se parte del supuesto de que todos los profesores están alineados con el ideario del colegio en temas vinculados con la educación y la conducta sexual de los escolares.

No, la verdad es que asi plan establecido no, no hay; hay una linea, digamos, y es formación que se les da sobre todo en las clases, en las clases de Religión; pero así clases específicas de Educación Sexual los niños no tienen [...] cada vez que nosotros tenemos que hacer una unidad, ya sea porque hay un embarazo, ya sea porque tenemos conductas de riesgo. La psicóloga del colegio arma esta unidad para que el profesor pueda aplicarla [...], solamente en las unidades que necesitamos $y$ en que hemos visto necesidad $y$ en la hora de orientación. (E5, 36-47)

[...] no tenemos un proyecto que diga educación sexual que hacemos en el colegio, porque está tan metido en el colegio que, claro, por eso te decía, si tú quieres algo más (nombra a la encargada), quizás te dará algo de Catequesis, porque ella da charlas, de repente, pero si tú me dices ¿esa es la educación sexual del colegio?, no, es mucho más, es mucho más amplia. $(E 2,48)$
La otra modalidad considera un programa específicamente creado para abordar de forma sistemática y gradual los contenidos de educación sexual. En algunos casos, estos programas están prediseñados y forman parte de la oferta ministerial, los que a su vez pueden ser complementados y/o adaptados a partir de programas específicamente creados por especialistas en el tema, a la luz de las directrices del Magisterio de la Iglesia y del proyecto educativo de la institución. En otros casos, el programa ha sido construido por la misma institución educacional.

Los principios identificados por los entrevistados, que sustentan los distintos programas, son el desarrollo integral y la valoración de la persona como un todo, de modo que observen una clara complementariedad entre sexualidad y afectividad, estén basados en estudios científicos y orientados por los principios y valores cristianos. Según la descripción de los entrevistados, estos programas consideran una visión amplia e integrada de la persona y se caracterizan por contar con un plan estratégico de implementación transversal en el currículum, con metas para cada año y nivel, que se abordan de forma simultánea en distintas asignaturas y son monitoreados a través de distintos instrumentos de evaluación.

[...] tenemos un proyecto intencionado, de cinco años, que tiene un plan estratégico, que tiene metas por año, que las visitas se van a monitorear, que tenemos instrumentos de evaluación, que estamos levantando información, que estamos cualificando a los profesores, que los profesores hacen una reflexión trimestral sobre el trabajo, que hay revisión de las fichas de trabajo de los cuadernos de tutoría, [de modo] que con todo eso yo tengo toda la esperanza de que el impacto va a ser significativo, aquí, y toda la creencia de que va a ser asi; ahora, si no lo hacemos como yo te lo acabo de describir, seguramente no va a ser significativo, va a ser hablar una vez más del tema. $(E 1,254-260)$ 
Otra característica descrita por los entrevistados es que los profesores reciben capacitación participando en diferentes actividades vinculadas con la actualización de los contenidos. Incluso algunos dicen contar con supervisión en la práctica pedagógica. Entre estas actividades mencionan jornadas trimestrales y/o anuales, reuniones semanales, observación retroalimentada de clases.

\section{Personas involucradas en su ejecución: alumnos, familias y profesores}

La implementación de las distintas modalidades a través de las que se imparte la educación sexual a los estudiantes considera tres actores claves: los alumnos, las familias y los profesores. Respecto de los estudiantes, los entrevistados reconocen en ellos ciertas características que intervienen en la forma y contenido que se les entrega en materia de educación sexual. Entre ellos, la iniciación sexual temprana y trayectorias sexuales distintas y heterogéneas en personas del mismo grupo etario, sin cuestionamientos personales respecto de su sexualidad, en un contexto en que se estimula la "cultura de lo desechable", siendo la sexualidad parte de los bienes que pueden tomarse y desecharse a voluntad. Además, se reconoce en ellos cierto desprecio por la ayuda que pudieran recibir de los profesores, con la creencia de saberlo todo, pese a que los entrevistados observan una gran necesidad de formación y acompañamiento.

En relación con la familia, esta es considerada como el agente socializador y formador por excelencia en este tema y, en ese sentido, los colegios cumplirían un rol complementario. Por lo mismo, la situación ideal es que exista una alianza entre la familia y el colegio para que los estudiantes alcancen una formación integrada y significativa. Los entrevistados reconocen dificultades al respecto, ya que hay familias que delegan al colegio esta labor como responsables únicos, y se observa escaso compromiso con la educación de sus hijos y poca participación en instancias formativas. Por otro lado, de acuerdo con los entrevistados, algunas familias consideran que la educación sexual no debería ser tratada en el colegio, ya que a las instituciones educacionales les correspondería preocuparse del rendimiento académico, más que de la formación personal de sus hijos.

[...] prefieren pasarnos la pelota, asi, de frente, o sea, como [si dijeran:] "si lo viste en el colegio, entonces yo no lo hablo, porque, como es un tema dificil de tocar, mejor no lo toco" [...]. Otras familias nos han dicho: "por qué ustedes se meten en estas áreas". (E1, 218-229)

Ante esta realidad, algunos colegios optan por excluir del todo a la familia y desarrollar su tarea en un proceso paralelo. Otros, en cambio, plantean distintas estrategias que se han implementado en función de formar una alianza con los padres/tutores/familiares de los estudiantes como forma de potenciar el trabajo a partir de discursos comunes, sobre todo en establecimientos que cuentan con programas de educación sexual estructurados, tales como reuniones de difusión del programa del colegio en materia de educación sexual, cursos de orientación familiar para padres, obligatorios y periódicos, a cargo de profesionales externos al colegio y vinculados al área (psiquiatras, orientadores, etc.). Los entrevistados reconocen ingenuidad e ignorancia de los padres y una gran necesidad de formación de las familias en materia de educación sexual. En relación con la implementación de los programas, en general, en instituciones con o sin un programa establecido destaca la heterogeneidad de los encargados. Entre ellos, psicólogos, orientadores, directivos del colegio, profesores en función de tutoría y profesores de asignatura, especialmente de Biología y/o Religión y la alta rotación de profesores que deben asumir la tarea.

Respecto de los profesores, los entrevistados destacan que, en general, los colegios no describen un perfil de ingreso relacionado con la formación en afectividad y sexualidad y tampoco lo consideran como requisito para su contratación; sin embargo, 
se les pide validar y promover la línea de valores del colegio, lo que muchas veces les provoca un exceso de tensión: "pero no se les pide formación, porque cuando empezamos a pedir formación nos va quedando como todo el mundo afuera" (E4, 92-93).

Los entrevistados plantean que muchos profesores se sienten incómodos, ya que perciben cierta censura por parte del colegio respecto de ideas personales relacionadas con la sexualidad en oposición a los planteamientos del colegio, las que se verían afectadas por su visión, creencias y la forma en que han vivido sus propias historias vinculadas al tema: "es que por las propias creencias o por las propias historias personales, no conectar con la necesidad, con las inquietudes del mundo adolescente o el mundo de tus educandos, eso puede ser una dificultad importante" (E7, 359-361).

Por otro lado, muchos profesores reconocen que prefieren no abordar temas de sexualidad, ya sea porque les produce temor tratarlos o porque "se sienten incómodos". En algunos casos esto se debería a que se perciben desconectados del mundo juvenil, en otros a la falta de formación, lo que les dificultaría la posibilidad de abordar mitos e inquietudes de estudiantes, lo que, sumado al gran número de escolares en el aula, hace más difícil crear un clima de acogida que permita conocer a los estudiantes, identificar sus necesidades y responder en forma significativa a sus inquietudes.

\section{Discusión}

De acuerdo con lo expresado por los informantes, se develan claramente diversas tensiones cuando se trata de analizar el desafío de la educación de sexual en el contexto de colegios católicos, insertos en nuestro tiempo y cultura. Considerando que la sexualidad es parte integral de la vida de las personas y que, según la Organización de las Naciones Unidas para la Educación, la Ciencia y la Cultura (Unesco), ella contribuye al desarrollo de la identidad y al desarrollo social (Unesco, 2014), es pertinente analizar los resultados desde múltiples perspectivas. En esta dirección, los resultados obtenidos se pueden estructurar en cuatro ejes claves: 1) perspectiva de la salud pública; 2) medición del impacto de los programas de educación sexual; 3) perspectiva de los docentes a cargo de la enseñanza en programas de educación sexual; y 4) enseñanza de la sexualidad desde la perspectiva de los valores católicos.

\section{Perspectiva de la salud pública}

La Encuesta Nacional de la Juventud muestra que ha ido disminuyendo la edad de iniciación sexual en los adolescentes en Chile. La encuesta 2012 mostró que el $48 \%$ de los jóvenes entre 15 y 19 años era sexualmente activo. La sexualidad ya no se asocia al matrimonio o a la reproducción y el número de parejas sexuales ha aumentado, siendo de 1,6 el promedio de parejas sexuales en los últimos 12 meses (1,8 en hombres y de 1,3 en mujeres). Por otra parte se observa un aumento sostenido del contagio de $\mathrm{VIH}$, siendo el grupo etario de 10 a 19 años el que presenta el mayor porcentaje de ascenso (Instituto Nacional de la Juventud, 2012).

En relación con el embarazo adolescente, la tasa se ha mantenido relativamente estable en los últimos 15 años, con alrededor de 155 nacimientos por cada mil nacidos vivos (15\% del total de embarazos). Desde el año 1998 al 2009 se produjo un aumento leve pero sostenido, que se revirtió desde el año 2010 al 2013 (Ministerio de Salud, 2013). La tasa de embarazo adolescente es, en general, más alta en comunas de menor ingreso socioeconómico; sin embargo, sus causas son multifactoriales, con influencia de componentes económicos, sociales y culturales (Ministerio de Salud, 2013). El embarazo adolescente se asocia a mayor morbilidad y mortalidad materna, a un aumento en el riesgo de deserción del sistema educacional, de inserción precaria en el mercado laboral y de formación de hogares uniparentales con mayor vulnerabilidad social y económica, lo cual reproduce inequidades sociales importantes (Organización Panamericana de la Salud, 2007). 
A nivel mundial se han desarrollado muchas estrategias/programas orientadas principalmente a la prevención del embarazo adolescente y del contagio de enfermedades de transmisión sexual $(\mathrm{VIH} /$ SIDA y otras). Una revisión sistemática de la Cochrane Collaboration sobre estrategias para prevenir el embarazo adolescente no deseado encontró 101 ensayos controlados randomizados sobre el tema, de los cuales 41 fueron incluidos, con un total de 95.662 participantes entre 9 y 24 años, la mayoría de países desarrollados (de EE.UU., principalmente). Se estudiaron tres tipos de intervenciones: educación sexual, promoción de métodos anticonceptivos e in tervenciones múltiples, que combinaban las dos anteriores. Los resultados mostraron que las intervenciones múltiples señalan una pequeña diferencia, aunque significativa, en la disminución de embarazos no deseados. No se evidenciaron diferencias en la edad de inicio de la actividad sexual, en el uso de métodos anticonceptivos (preservativos $\mathrm{u}$ hormonales) y tampoco en la prevalencia de enfermedad de transmisión sexual o el porcentaje de personas en abstinencia. Las intervenciones que promovieron el uso de anticoncepción no mostraron efectos en su objetivo de reducir el embarazo. En relación con las intervenciones educativas solas, los datos no fueron insuficientes para obtener conclusiones válidas (Oringanje et al., 2009).

\section{Medición del impacto de los programas de educación sexual}

Otra reflexión surge de los programas de formación y focaliza la escasa medición del impacto o la efectividad que estos tienen en los colegios estudiados. En los resultados obtenidos se observa que ninguno de los colegios participantes había desarrollado la medición del impacto de la aplicación de sus programas de educación sexual, ya sea en actitudes, en conocimientos o en habilidades de los alumnos o de los profesores.

En el ámbito mundial hay poca claridad sobre cuál estrategia es la más efectiva o indicada. La re- visión de Cochrane Collaboration, ya mencionada, muestra que, pese a que la educación sexual es una práctica habitual en muchos países, hay poca evidencia de su real impacto desde una perspectiva integral (Unesco, 2014). En Chile tampoco existe medición del impacto de la implementación de esta política y no se dispone de estudios que reporten los programas en su conjunto; solo se tiene la evaluación que los autores han realizado de algunos de ellos (Cabezón et al., 2005; Vigil et al., 2005; Toledo et al., 2000). En el caso del Programa Teen Star, las evaluaciones prospectivas de doble ciego randomizadas, realizadas por el propio equipo a cargo de la intervención, muestran que se logra disminuir la tasa promedio anual de embarazo adolescente de $4,87 \%$ a $0,87 \%$ y que los alumnos adquieren herramientas que logran reducir conductas como la promiscuidad sexual y el inicio de relaciones sexuales a temprana edad (Cabezón et al.., 2005; Vigil et al., 2005).

Finalmente, parece haber consenso en que la búsqueda de una educación de calidad involucra y requiere de una estrecha relación entre distintos actores, padres y apoderados, niños y jóvenes y educadores, los que aparentemente se encuentran tensionados entre sí de acuerdo con los resultados de este estudio.

\section{Perspectiva de los docentes a cargo de programas de educación sexual}

Tal como se mencionó, Cohen et al. (2012) destacan cuatro elementos directamente relacionados con la percepción que tienen los docentes a cargo de programas de educación sexual, desde una visión cristiana, con el grado de efectividad que puede alcanzar su enseñanza. Estos son los conocimientos sobre la materia, el nivel de conformidad con los principios que se enseñan, el entrenamiento en la enseñanza de dicho contenido y el grado de vivencia existencial del docente con la enseña impartida, es decir, la coherencia de vida. Respecto de los conocimientos sobre la materia, parece claro que no se podría enseñar lo que no se sabe. Este elemento 
se detecta como foco de análisis desde la formación inicial de los educadores. Darling-Hammond y Bransford (2005), buscando el consenso sobre los elementos claves de un currículum de formación inicial de profesores, plantean que las interacciones entre profesores, estudiantes y contenidos pueden organizarse dentro de la enseñanza como profesión, entre el conocimiento de los aprendices y su desarrollo en contextos sociales, el conocimiento de los contenidos y objetivos del currículum y, por último, el conocimiento de la enseñanza. En la intersección de estas tres áreas se encuentra la visión de la práctica profesional.

En materia de educación sexual, los informantes del estudio declaran la tensión entre el conocimiento de la materia que tienen los estudiantes y los contenidos que se pretende entregar. En este sentido, la Unesco (2014) plantea que la educación sobre sexualidad va más allá de la adquisición de conocimientos, ya que proporciona competencias y habilidades para la vida y forma parte de la educación fundamental. Desde esta perspectiva, se comprenden la tensiones emergentes en el análisis de los informantes del estudio, ya que no solo se trata de entregar a los jóvenes una sólida formación académica, sino también de proporcionarles conocimientos y habilidades para vivir expresando el máximo de su potencial personal, para el logro de su bienestar físico, psicológico y emocional (Unesco, 2014).

Segundo, respecto de la conformidad con los principios que se enseñan, podría leerse la dificultad de enseñar aquello en lo que no se cree. Desde esta perspectiva, la tensión entre la entrega de contenidos y la formación de personas tendría su raíz en la identidad misma del rol de profesor, entendida como la forma como los profesionales definen y asumen las tareas que les son propias y el modo como entienden sus relaciones con otras personas que cumplen las mismas tareas (Ávalos y Sotomayor, 2012).

Un estudio realizado en Chile con una muestra representativa nacional de alrededor de 1990 docentes solicitó a los profesores indicar las razones que tuvieron para elegir la docencia. Una de las conclusiones que el estudio plantea es que

[...] la descripción identitaria de los docentes chilenos concuerda con lo que podría considerarse como el retrato tradicional de la misión de los educadores [...]. Los profesores y profesoras chilenos definen su identidad en referencia directa a sus alumnos, a quienes deben enseñar contenidos específicos mandatados por el sistema educacional y, al mismo tiempo, educar como personas y miembros de la sociedad, desarrollando en ellos todas sus capacidades intelectuales, afectivas y sociales. (Ávalos y Sotomayor, 2012, p. 79)

En este mismo estudio, los profesores identifican las complejidades de este rol: alumnos cada vez más críticos y exigentes que demandan tiempos de formación, planificación y evaluación a un docente con un bajo estatus social y condiciones difíciles de trabajo, a pesar de lo cual la apreciación general de la identidad recogida en las percepciones de los profesores del estudio sigue siendo positiva. En este sentido, se podría analizar que las dificultades develadas en los profesores frente a la educación de la sexualidad en colegios católicos es un reflejo de los desafíos propios de la labor docente.

Tercero, en relación con el entrenamiento en la enseñanza de dicha materia, los resultados del estudio plantean la necesidad de un entrenamiento entendido como un procedimiento pensado para obtener conocimientos, habilidades y capacidades. Estos conocimientos, habilidades y capacidades se orientan al cómo trasmitir el contenido que se enseña. Al respecto, parece pertinente recoger el aporte de Lee Shulman (2005), quien plantea que la investigación sobre la enseñanza se ha realizado en forma genérica, con prescindencia de la materia que se va a enseñar, es decir, sin considerar el cómo se enseña una determinada materia, lo que conoce como "paradigma ausente". Este concepto del conocimiento peda- 
gógico del contenido define y desarrolla algunas de las dificultades expresadas por los entrevistados en el estudio (Shulman, 1987, 2001).

El cuarto y último punto mencionado por Cohen et al. (2012) es el grado de vivencia existencial del docente con la enseña impartida, es decir, la coherencia de vida. En este sentido, el análisis de los resultados va en la línea de las dificultades de "enseñar lo que no se es". Al respecto, los entrevistados explicitan la tensión entre sus propias creencias, sus experiencias de vida y lo que deben enseñar. En relación con esto último, son iluminadoras las palabras de Benedicto XVI en su carta a los educadores de Roma, que plantea:

[...] asi pues, la educación no puede prescindir del prestigio, que hace creíble el ejercicio de la autoridad. Es fruto de experiencia y competencia, pero se adquiere sobre todo con la coherencia de la propia vida y con la implicación personal, expresión del amor verdadero. Por consiguiente, el educador es un testigo de la verdad y del bien; ciertamente, también él es frágil y puede tener fallos, pero siempre tratará de ponerse de nuevo en sintonía con su misión. (Benedicto XVI, 2008).

En síntesis, el análisis de la perspectiva de los docentes a cargo del desarrollo de programas de educación sexual señala tensiones que no solo son propias de esta área del contenido a enseñar y que, sin considerar las particularidades propias de su naturaleza, implican todas las tensiones y desafíos del saber pedagógico, es decir, qué y cómo se enseña desde la identidad del ser y pensar del educador.

\section{Enseñanza de la sexualidad desde la perspectiva de los valores católicos}

Desde la perspectiva de los valores cristianos católicos, la sexualidad y su educación no ha dejado de ser algo que colinda con lo sagrado. En efecto, esto es así porque el hombre es una creatura, es decir, una realidad que hace referencia a un Creador. Y en este marco el respeto es el valor crucial que rige la unión entre un hombre y una mujer. En efecto, el respeto es un presupuesto para entender y captar los valores y una parte central de la adecuada respuesta al valor. De ahí que represente una condición necesaria $y$, al mismo tiempo, un elemento esencial de todas las virtudes (Von Hildebrand, 2004). Esta realidad en el plano ético de la persona tiene un rol fundamental en la vida sexual entre un hombre y una mujer. Así, el respeto juega un papel especial en el reino de la pureza. La castidad supone esencialmente una actitud respetuosa en relación con el secreto del amor entre el hombre y la mujer, una conciencia que impregna la esfera de lo sexual con santo recato, y a la que debiera uno aproximarse solamente con la expresa sanción de Dios. La castidad es incompatible con una actitud general presuntuosa frente a lo existente, ya asuma un carácter frívolo o cínico, ya pueda convertirse en una aproximación íntima, obtusa, ingenua y pagada de sí misma respecto a los secretos del cosmos. La castidad exige estima a la persona amada y a su cuerpo y profundo respeto a la honda y misteriosa unidad de dos almas en una sola carne, así como el misterio del alumbramiento de una nueva persona (Von Hildebrand, 2004).

El Magisterio de la Iglesia Católica también ayuda a comprender la esencia del sacramento del matrimonio cristiano, en tanto que como

[...] comunión de amor entre Dios y los hombres, contenido fundamental de la Revelación y de la experiencia de fe de Israel, encuentra una significativa expresión en la alianza esponsal que se establece entre el hombre y la mujer. En virtud de la sacramentalidad de su matrimonio, los esposos quedan vinculados uno a otra de la manera más profundamente indisoluble. El amor conyugal comporta una totalidad en la que entran todos los elementos de la persona - reclamo del cuerpo y del instinto, fuerza del sentimiento y de la afectividad, aspiración del espiritu y de la voluntad-; mira a una unidad profundamente personal, que más allá de la unión en una sola 
carne, conduce a no hacer más que un solo corazón y una sola alma; exige la indisolubilidad y fidelidad de la donación reciproca y definitiva y se abre a la fecundidad. Según el designio de Dios, el matrimonio es el fundamento de la comunidad más amplia de la familia, ya que la institución misma del matrimonio y del amor conyugal están ordenados a la procreación y educación de la prole, en la que encuentran su coronación. (Juan Pablo II, 1981)

En relación con el acto conyugal, el papa Pablo VI (1968), en su encíclica Humanae Vitae afirmó que "la doctrina de la Iglesia está fundada sobre la inseparable conexión que Dios ha querido y que el hombre no puede romper por propia iniciativa entre los dos significados del acto conyugal: el significado unitivo y el significado procreador", recalcando como acción intrínsecamente deshonesta "toda acción que, o en previsión del acto conyugal, o en su realización, o en el desarrollo de sus consecuencias naturales, se proponga como fin o como medio, hacer imposible la procreación".

Sin embargo, en la práctica la educación en la sexualidad basada en valores cristianos se ve amenazada por grandes cambios culturales y sociológicos. Por una parte, existe un proceso de secularización que tiende a reducir la fe y la Iglesia al ámbito de lo privado. Junto con eso, al negar toda trascendencia, se ha producido una deformación ética, con un debilitamiento de la noción de pecado personal y social y un aumento del relativismo, que muchas veces se asocia a desorientación, especialmente en la adolescencia. "Estamos en una sociedad de la información, que nos satura de datos, pero que nos lleva a una gran superficialidad a la hora de plantear cuestiones morales" (Francisco I, 2013). En el plano social, se observa una crisis antropológica. El papa Francisco añade que "el primer lugar está ocupado por lo exterior, lo inmediato, lo visible, lo rápido, lo superficial, lo provisorio. Lo real cede lugar a la apariencia" (2013). En relación con la familia, precisa que
[...] la familia atraviesa una crisis cultural profunda, como todas las comunidades y vínculos sociales, pero en su caso, la fragilidad de los vínculos es especialmente grave porque se trata de la célula básica de la sociedad, el lugar donde se aprende a convivir en la diferencia y a pertenecer a otros, $y$ donde los padres trasmiten la fe a sus hijos. El matrimonio es visto como una mera gratificación afectiva que puede constituirse de cualquier manera y modificarse de acuerdo con la sensibilidad de cada uno. (Francisco I, 2013)

A nivel cognitivo se observa fragmentación, lo cual constituye una amenaza para encontrar la coherencia y el significado profundo de la vida. Existen muchísimos conocimientos, pero falta la visión sistémica, de conjunto. Junto con esto se observa una amenaza a la necesaria firmeza en el actuar, con consecuencias tales como evasión del compromiso o impulsividad (Giovanelli, 2012). Se evidencia una pérdida del sentido de educar y la dificultad para realizarlo. El papa Benedicto XVI afirma que "se habla de una gran 'emergencia educativa' confirmada por los fracasos en los que muy a menudo terminan los esfuerzos por formar personas sólidas, capaces de colaborar con los demás y de dar un sentido a su vida" (2008).

\section{Conclusión}

El gobierno de Chile, desde el año 2010, a través de la Ley 20418 promueve programas de educación sexual escolar, cuya intención es disminuir la tasa de embarazo adolescente, retrasar el inicio de la actividad sexual e informar y formar a los jóvenes y niños en esta materia. A partir de esto, la mayoría de los colegios en Chile y asimismo los establecimientos católicos implementan programas de educación sexual. Este estudio pone de manifiesto la escasez de trabajos científicos sobre educación desde la perspectiva católica o cristiana. Asimismo se observa que, si bien se han implementado diversos programas sobre educación sexual en Chile, estos han sido 
poco evaluados respecto de su impacto y su efectividad, e igual ocurre con respecto a las políticas públicas en esta materia.

De los resultados se destacan las principales dificultades encontradas en el proceso educativo en torno a la educación en la afectividad y sexualidad. Hay tensión entre los postulados de la doctrina católica, las vivencias de los jóvenes y las creencias de las familias y de los profesores, los cuales muchas veces se contraponen. La falta de participación de las familias hace que deleguen la educación sexual principalmente en el colegio y no asuman en forma plena su rol educativo en esta materia. La dificultad operativa de impartir esta enseñanza está dada por la falta de tiempo protegido, de infraestructura adecuada, de profesores capacitados y de coherencia de vida entre lo que se vive y lo que imparte. Finalmente, hay que subrayar la importancia del contexto en el cual viven los jóvenes actuales, caracterizado por estar erotizado, por la cultura de lo desechable y de la satisfacción inmediata, con acceso ilimitado a información en los medios de comunicación y redes sociales, etc., elementos que continuamente atentan contra la vivencia de una vida al alero de valores cristianos.

A partir de este trabajo surgen algunas recomendaciones: a) la selección de profesores u orientadores que imparten esta materia debe considerar que posean formación adecuada y coherencia de vida; b) los colegios deben implementar programas de formación permanente para profesores en esta materia, tanto en contenidos como en nuevas metodologías; y c) es necesario establecer acciones orientadas a evaluar la efectividad de las políticas y de los programas de educación sexual. Dentro las limitaciones de esta investigación se deben considerar que los resultados obtenidos son el reflejo exclusivo de la vivencia de un grupo de orientadores, profesores o directivos de colegios de la Región Metropolitana de Chile y que, si bien comparten elementos con otros estudios en esta línea, los hallazgos pueden no ser generalizables.

\section{Referencias}

Ávalos, B. y Sotomayor, C. (2012). Cómo ven su identidad los docentes chilenos. Revista Perspectiva Educacional, 57(1), 77-95.

Aschcraft, C. (2012). But how do we talk about it? Critical literacy practices for addressing sexuality with youth. Curriculum Inquiry, 42(5), 597-628.

Benedicto XVI (2008). Mensaje a la diócesis de Roma sobre la tarea urgente de la educación. Librería Editrice Vaticana.

Benedicto XVI (2009). Caritatis in veritatis. Librería Editrice Vaticana. Recuperado de http://w2.vatican.va/content/benedict-xvi/es/ency clicals/documents/hf_ben-xvi_enc_20090629_caritas-in-veritate.pdf

Bertelsen, R. (2004). Libertad de enseñanza: dos sentencias del Tribunal Constitucional. En Buchheister, A., Fermandois, A. y Soto, S. (eds.), Sentencias destacadas 2004 (pp. 157-193). Santiago: Instituto Libertad y Desarrollo. Recuperado de http://lyd.org/wp-content/uploads/2015/o2/pp-157-193-Libertad-deense\%C3\%B1anza-dos-sentencias-del-Tribunal-Constitucional-RBertelsen.pdf

Bruckner, H. y Bearman, P. (2005). After the promise: The STD consequences of adolescent virginity pledges. Journal of Adolescent Health, 36(4), 271-278. 
Cabezón, C., Vigil, P., Rojas, I., Leiva, M., Riquelme, R., Aranda, W. y García, C. (2005). Adolescent pregnancy prevention: an abstinence-centered randomized controlled intervention in a Chilean public high school. Journal of Adolescent Health, 36(1), 64-69.

Cohen, J., Byers, S. y Sears, H. (2012). Factors affecting Canadian teachers' willingness to teach sexual health education. Sex Education, 12(3), 299-316.

Darling-Hammond, L. y Bransford, J. (2005). Preparing teachers for a changing world. What teachers should learn and be able to do. California: Jossey-Bass Education Series.

Flick, U. (2004). Introducción a la investigación cualitativa. Madrid: Morata.

Francisco I (2013). Exhortación apostólica Evangelii Gaudium. Santiago: Ediciones Paulinas.

Freedman-Doan, C. R., Fortunato, L. Henshaw, E. J.y Titus, J. M. (2013). Faith-based sex education programs:What they look like and who uses them. Journal of Religious Education, 52(1), 247-262.

Giovanelli, F. (2012). Conferencia: ¿Está hoy la persona humana en el corazón del desarrollo? En La persona en el corazón del desarrollo: una mirada desde el pensamiento actual. Santiago. Chile: Publicaciones Pastoral UC.

Glaser, B.y Strauss, A. (2010). The Discovery of Grounded Theory: Strategies for Qualitative Research. Nueva Jersey: Aldine Transaction.

Gobierno de Chile (2012). Historia de la Ley No. 20418. Fija normas sobre información, orientación y prestaciones en materia de regulación de la fertilidad. Documento Oficial, 28 de enero. Biblioteca del Congreso Nacional de Chile.

Instituto Nacional de la Juventud (2012). Séptima Encuesta Nacional de la Juventud. Chile: Ministerio de Desarrollo Social. Recuperado de http://www.injuv.gob.cl/portal/wp-content/files_mf/septimaencuestanacionaljuventud2.pdf

Juan Pablo II (1981). Exhortación apostólica Familiaris Consortio: sobre el matrimonio y la familia cristiana. Santiago: Ediciones Paulinas.

Juan Pablo II (1985). Carta apostólica a los jóvenes y a las jóvenes del mundo, con ocasión del XXX Año Internacional de la Juventud. Madrid: Biblioteca de Autores Cristianos.

Kirby, D. (2002). Adolescent unprotected sex, pregnancy, and childbearing. Journal of Sex Research, 39(1), 51-57.

Ley 19638 (1999). Diario Oficial, 14 de octubre. Chile.

Ley 20418 (2010). Diario Oficial, 28 de enero. Chile.

Luquis, R. R., Brelsford, G. M. y Rojas-Guyler, L. (2011). Religiosity, spirituality, sexual attitudes, and sexual behaviors among college students. Journal of Religious \& Health, 51(3), 601-614. 
ISSN 0123-1294 | e-ISSN 2027-5358 | Educ.Educ. Vol. 20. No. 3 | Septiembre-diciembre de 2017 | pp. 343-363.

Universidad de La Sabana | Facultad de Educación

Madero, C. (2012). La escuela católica en un escenario desigual. Revista Mensaje, 61(611), 38-41.

McKinney, S. J. y Sullivan, J. (2013). Education in a Catholic perspective. Surrey: Ashgate.

Ministerio de Educación (2012). Orientaciones para el diseño e implementación de un programa en sexualidad, afectividad y género. Recuperado de http://portales.mineduc.cl/usuarios/convivencia_escolar/ doc/201307221719120.PDF_orientaciones_sexualidad.pdf

Ministerio de Educación, Servicio Nacional de la Mujer (2011). Portafolio de Programas de Educación Sexual. Recuperado de https://portal.sernam.cl/?m=sp\&i=377 www.mineduc.cl

Ministerio de Educación (2012). Planes de Estudio. Recuperado de http://www.mineduc.cl/index5_int.php?id_ portal $=47 \&$ id_contenido $=17116 \&$ id_seccion $=3264 \& c=346$

Ministerio de Salud (2013). Situación actual del embarazo adolescente en Chile. Recuperado de http://web. minsal.cl/portal/url/item/c908a2010f2e7dafeo40010164010db3.pdf

Organización Panamericana de la Salud (2007). Salud en las Américas. Recuperado de http://www.paho.org/ HIA/index.html

Oringanje, C., Meremikwu, M. M., Eko, H., Esu, E., Meremikwu, A. y Ehiri, J. E. (2009). Interventions for preventing unintended pregnancies among adolescents. The Cochrane database of systematic reviews, 7(4), CDo05215. Recuperado de http://onlinelibrary.wiley.com/doi/10.1002/14651858.CDo05215.pub2/abstract;j sessionid=D7363F95FBAB1F12357336739ADFE1A5.fo4to2

Pablo VI (1968). Carta encíclica Humanae Vitae: sobre la regulación de la natalidad. Roma: Ediciones Vaticano.

Patton, M. (1990). Qualitative evaluation and research methods. California: Sage.

Peterson, C. y Seligman, M. E. P. (2004). Character strengths and virtues: A handbook and classification. Nueva York: Oxford University Press.

Pío XI (1930). Carta encíclica Casti Connubii: acerca del matrimonio cristiano. Roma: Ediciones Vaticano.

Rector, R. (2002). The effectiveness of abstinence education programs in reducing sexual activity among youth. The Heritage Foundation, report Education, 8 de abril. Recuperado de http://www.heritage.org/research/ reports/2002/04/the-effectiveness-of-abstinence-education-programs

Regnerus, M. (2007). Forbidden fruit: Sex and religión in the lives of American teenagers. Oxford: Oxford University Press.

Sandoval, C. (1996). Investigación cualitativa. Bogotá: Arfo. Recuperado de https://docs.google.com/viewer$? \mathrm{a}=\mathrm{v} \&$ pid $=$ sites\&srcid=ZGVmYXVsdGRvbWFpbnxjdWFsaXRhdGl2YXVuaWNvcmR8Z3g6MWZIYTk4MW NjOGU4ODUwNw

Shulman, L. (1987). Knowledge and teaching: foundations of the New Reform. Harvard Educational Review, 57(1), pp. 1-22. 
Shulman, L. (2001). Conocimiento de la enseñanza. Estudios Públicos, 83,163-196.

Shulman, L. (2005). El saber y entender de la profesión docente. Estudios Públicos, 99, 195-224.

Stein, M. K.y Wang, M. C. (1988). Teacher development and school improvement: The process of teacher change. Teaching and Teacher Education, 4(2), 171-187.

Strauss, A. y Corbin J. (1998). Basics of Qualitative Research. Techniques and Theory for Developing Grounded Theory. Londres: Sage.

Sultmann, W. y Brown, R. (2014). Catholic school identity and the new evangelization. Journal of Religious Education, 62(1), 1-13.

Toledo, V., Luengo, X., Molina, R., Murray, N., Molina, T. y Villegas, R. (2000). Impacto del programa de educación sexual: Adolescencia tiempo de decisiones. Revista de la Sociedad Chilena de Obstetricia y Ginecología Infantil y de la Adolescencia, 7(3), 73-86.

Unesco (2014). Educación integral de la sexualidad: conceptos, enfoques y competencias. Santiago: Orealc/ Unesco. Recuperado de http://unesdoc.unesco.org/images/o023/002328/23280oS.pdf

http://unesdoc.unesco.org/images/0023/002328/23280oS.pdf

Vigil, P., Riquelme, R., Rivadeneira, R. y Aranda, W. (2005). TeenStar: una opción de madurez y libertad. Programa de educación integral de la sexualidad, orientado a adolescentes. Revista Médica de Chile,133(10), 1173-1182.

Von Hildebrand, D. (2004). La importancia del respeto en la educación. Educación y educadores, 7, 221-228.

Whitehead, B. L. (2001). What does God have to do with teen pregnancy prevention? En B. D. Whitehead, Wilcox, L. y Rostosky, S. (eds.), Keeping the faith: The role of religion and faith communities in preventing teen pregnancy (pp. 1-30). Washington, DC: The National Campaign to Prevent Teen Pregnancy.

Wojtyla, K. (1960). Amor y responsabilidad. España: Palabra. 